\title{
ANKLE DYNAMIC IN STROKE PATIENTS: AgONIST VS. ANTAgONIST MUSCLE RELATIONS
}

\author{
Augusta Silva ${ }^{1,2,3}$, Andreia S. P. Sousa ${ }^{1,2,4}$, JoÃo Manuel R. S. TAVAres ${ }^{4}$, AnA \\ TinOCO, RUBIM SANTOS ${ }^{2}$, FILIPA SOUSA ${ }^{3}$ \\ ${ }^{1}$ Área Científica de Fisioterapia, Escola Superior de Tecnologia da Saúde do Instituto Politécnico do Porto, Vila \\ Nova de Gaia, Portugal.smaugusta@gmail.com,asp@estsp.ipp.pt \\ ${ }^{2}$ Centro de Estudos de Movimento e Actividade Humana (CEMAH), Escola Superior de Tecnologia da Saúde do \\ Instituto Politécnico do Porto, Vila Nova de Gaia, Portugal. rss@estsp.ipp.pt \\ ${ }^{3}$ Laboratório de Biomecânica do Porto, Faculdade de Desporto, Universidade do Porto, Porto, Portugal. \\ filipas@fade.up.pt \\ ${ }^{4}$ Instituto de Engenharia Mecânica e Gestão Industrial, Departamento de Engenharia Mecânica, Faculdade \\ de Engenharia, Universidade do Porto,Porto, Portugal.tavares@fe.up.pt
}

\section{*Corresponding author:}

Área Científica de Fisioterapia, Escola Superior de Tecnologia da Saúde do Instituto Politécnico do Porto Rua Valente Perfeito, 322 4400-330, Vila Nova de Gaia, Portugal.

E-mail address: smaugusta@gmail.com (Augusta Silva).

Number of pages: 9

Number of tables: 2

Number of figures: 2

Declaration of interest: No conflicts of interest. 


\begin{abstract}
Introduction: Atypical ankle patterns of muscle activity during gait are commonly reported in patients with stroke. These findings can be due to changes between tibialis anterior (TA) and soleus (SOL) coactivation mechanisms. Objective: To compare the electromyographic activity (EMGa) of SOL and TA muscles and antagonist coactivation (C) level in the contralateral (CONTRA) and ipsilateral (IPSI) limbs to the side of the stroke lesion during stance phase of the gait cycle. Methods: Twelve subjects with a stroke episode participated in this study. The electromyographic signal of TA and SOL and ground reaction forces were acquired while subjects walked at their self-selected speed. Values of ground reaction forces were used to divide the stance phase of gait into initial contact, midstance and terminal stance. In each subphase the magnitude of TA and SOL was calculated as well as the level of the antagonist C. Results: Although no statistical differences were found, mean values of SOL EMGa were lower in the IPSI in all stance phases in relation to the CONTRA limb, and the opposite was observed in the TA EMGa. Moreover, higher mean levels of antagonist $\mathrm{C}$ were only found during initial contact sub-phase in CONTRA limb and in the other sub-phases in the IPSI limb. Besides, statistical differences were observed only during midstance. Conclusion: In stroke subjects the antagonist $\mathrm{C}$ level during midstance of gait my reflected the dysfunction of the neuronal system over the IPSI limb.
\end{abstract}

KEY WORDS: Stance phase of Gait, electromyography activity, soleus and tibial muscles, antagonist coactivation, stroke. 


\section{INTRODUCTION}

The ability to balance the activity of the lower limb muscles during human gait is required in order to promote the weight bearing capacity, stability and body orientation (Den Otter et al., 2007). An effective gait requires a complex interplay between rhythmic spinal neuronal activity, the influence of supraspinal structures and the polysynaptic processing inherent to the spinal neuronal activity (Mayer, 2002).

In stroke subjects, high levels of coactivation (C) between the agonist and antagonist muscles at the ankle joint have been described as an adaptative mechanism for postural stability (Busse et al., 2006; Higginson et al., 2006; Lamontagne et al., 2002; Lamontagne et al., 2000). The interest of studying the $\mathrm{C}$ mechanism between the agonist and antagonist muscles at the joint level is related to motor control issues (Schumway-Cook et al., 2007) and is a tool for detecting pathophysiological mechanisms (Frigo et al., 2009). To selected rehabilitation strategies in subjects with ankle muscle dysfunction (like stroke subjects), it is essential to define how these subjects deviate from normal patterns (Orendurff et al., 2005).

Along the different subphases of the stance phase of gait, the tibialis anterior (TA) and soleus (SOL) muscles change their role as agonist vs antagonist (Chmielewski et al., 2005; Darainy et al., 2008). In fact, when the stance phase is divided into a first subphase or initial contact, the TA acts as an agonist (Аyyappa, 1997; Bonell et al., 2007; Cappellini et al., 2006), with SOL in a lengthening position (Orendurff, et al., 2005). In the second sub-phase, also named midstance, the SOL muscle, assisted by the gastrocnemius, controls the rate of dorsiflexion of the ankle, keeping its action during the terminal stance to prepare the impulse of the foot (Ayyappa, 1997; Higginson, et al., 2006; Neptune et al., 2001; Orendurff, et al., 2005). Mayer (2002) states that in subjects with impaired gait due to injury of central nervous system (CNS), the changes in the reciprocal inhibition mechanisms mediated by Ia fibers are dependent on whether the muscle is the agonist or the antagonist of the movement. This analysis may allow the inference of the system predominantly affected, and thus a more efficient organization of the strategies used for rehabilitation.

Previous electromyographic based studies have been performed regarding the activity of the TA and medial gastrocnemius muscles in stroke subjects (Den Otter, et al., 2007; Lamontagne, et al., 2000). However, sub-cortical injuries located at the 
internal capsule level with dysfunction of the ventral-medial systems may justify changes in the activity of the ipsilateral (IPSI) SOL muscle. Likewise, the TA, under the control of the lateral cortico-spinal system (Capaday et al., 1999), presents changes in the contralateral (CONTRA) lower limb. As such, in this research, we aimed to study the electromyographic activity (EMGa) of these muscles also in IPSI limb (also referred as non-paretic side) in stroke subjects. Although there is low evidence to support the presence of a paretic type of muscle activation on IPSI limb (Lamontagne, et al., 2002), this hypothesis is supported by the neuronal connection of TA and SOL. Hence, the purpose of this study was to analyze the differences in SOL and TA muscle EMGa and antagonist C levels between CONTRA and IPSI limb in stroke subjects during the stance phase of gait.

\section{MeTHODS}

\subsection{Participants}

Twelve subjects with a first isquemic stroke, resulting in a hemiparesis, participated in this study (Table 1). Their injury was in the region of middle cerebral artery (MCA), more specifically in the internal capsule, which was confirmed by brain computerized axial tomography. All subjects included in the sample have a score lower to 34 on the Fugl-Meyer Assessment of Sensoriomotor Recovery After Stroke scale (Lamontagne et al. 2002) and the capacity to perform gait without the use of orthoses. The subjects have also preserved the cognitive function to understand orders, which was confirmed by assessment using the Mini-Mental State Examination. All potential subjects with previous history of neurologic pathology (e.g. Parkinson, pontine and cerebellar lesions), sensory impairment, diabetes, thrombophlebitis, history of lower limb surgery and any orthopedic or rheumatoid conditions interfering with sit to stand and stand to sit sequences were excluded. Subjects under medication that could affect the motor performance were also excluded. The clinical aspects that were taken into account, such as time evaluation, score of Fugl-Meyer Assessment of Sensorimotor Recovery and side lesion, are indicated in Table 1. All participants gave their informed consent according to the Declaration of Helsinki.

\subsection{Instruments}

For the evaluation of the lower limb was applied the corresponding part of the adapted version to the Portuguese population of the Fugl-Meyer Assessment of Sensorimotor Recovery 
After Stroke. The EMG signal was acquired and processed using the MP 150 Workstation model from Biopac Systems, Inc. (USA), and the Acqknowledge software (Biopac Systems, Inc., USA). The pairs of active silver chloride (AgCl) electrodes 150 B TDS circular surface were used having a diameter of $11.4 \mathrm{~mm}, 100 \mathrm{M} \Omega$ input impedance and 95dB CMRR (common-mode rejection ratio). A force plate, model FP4060-10 from Bertec Corporation (U.S.A), connected to a Bertec AM 6300 amplifier, with default gains, connected to a Biopac 16 bit analogical-digital converter was used to acquire the ground reaction force. The signals were acquired at $1000 \mathrm{~Hz}$.

\subsection{Procedures}

\subsubsection{Electrode placement}

Before the electrode placement, the skin was prepared to reduce the impedance to a level equal or inferior to $5 \mathrm{~K} \Omega$ (Camargos, Goulart and Teixeira-Salmela, 2009; Cheng et al. 2004; Correia \& Mil-Homens, 2004). The placement adopted was according to the SENIAM (Surface ElectroMyoGraphy for the Non-Invasive Assessment of Muscles) recommendations.

\subsubsection{Measurement}

After an explanation about the procedures, the subjects in barefoot and using shorts, were instructed to, after a voice command, perform gait at their usual speed, during a pre-defined distance. The usual speed was adopted since the plantar flexor muscles were the muscle group most used at this speed (Milot et al., 2008). It was ensured that during the task at least one complete stance phase was performed on the force plate. To prevent fatigue, one minute rest between each trial was provided and the necessary repetitions were performed in order to obtain three valid trials. The electromyographic data was acquired from both lower limbs simultaneously.

\subsubsection{Data analysis}

The raw EMG signal and the force plate data were processed using the Acqknowledge software. Hence, the raw EMG signal was filtered using a band-pass filter of $20 \mathrm{~Hz}$ and $500 \mathrm{~Hz}$ and processed using a root mean square (RMS) procedure (Billot et al., 2010; Lamontagne, et al., 2002; Lamontagne, et al., 2000). The signal from the force plate was also filtered, using a low pass filter of $10 \mathrm{~Hz}$, and the force values were normalized to the weight of each subject (Turns et al. 2007).

The stance phase was divided into three sub-phases using the force platform: 1) start of signal detection until the first peak of the ground attack, 2) between the peak ground attack to the second peak, 3) since the second peak until the end of the signal acquired. In each stance 
interval, the EMG magnitude and C values between TA and SOL (Darainy, et al., 2008) were evaluated. The $\mathrm{C}$ values were calculated according to:

$$
C(\%)=\frac{\text { antagonist activity }}{\text { agonist }+ \text { antagonist activity }} \times 100 \text {. }
$$

\subsection{Statistics}

Using descriptive statistics, measures of central tendency (mean) and dispersion (standard deviation) for the magnitude of TA and SOL's EMG activity were calculated. Regarding to the small sample size of both groups $(\mathrm{n}=10)$, it was assumed that the variables did not follow a normal distribution. Thus, the Wilcoxon Signed Rank Test was used to compare the magnitude of the muscles' activity and $\mathrm{C}$ between both lower limbs. The confidence interval used was of $95 \%$, with a significance level of 0.05 .

\section{RESULTS}

The curves shown in Figure 1 are representative of the muscles' activity of the subjects studied; mainly, for the raw EMG of TA and SOL muscles and of the vertical ground reaction force $(\mathrm{Fz})$ acquired during the stance phase of gait.

According to Figure 2, no statistical significant differences were observed in the EMGa between the two limbs. However, a descriptive analysis shown that mean values of the SOL EMGa were lower in the IPSI in all stance phases in relation to the CONTRA limb, and the opposite was observed in the TA EMGa, as this one presented lower values of EMGa in CONTRA in all stance sub-phases.

Although in all stance phases the mean of TA EMGa was lower in CONTRA limb, it was observed in this limb a higher level of the antagonist $\mathrm{C}$ value only during the initial contact subphase (Table 2). In the other sub-phases, the antagonist $C$ values were higher in the IPSI limb that presented a lower mean of SOL EMGa. However, only significant differences were found during midstance, where the IPSI limb presented a higher level of antagonist $\mathrm{C}$ in relation to the CONTRA limb.

\section{DISCUSSION}

After a stroke, the neuromuscular control of gait is unsettled either in the temporal organization and activation intensity of muscle activity (Den Otter, et al., 2007). An adequate ankle control during gait is important for normal gait pattern (Lin et al., 2006) and the antagonist $\mathrm{C}$ level may reflect the changes in the reciprocal inhibition mechanism in stroke subjects. The study of agonist vs antagonist relations may be useful to a better understanding of 
the mechanisms related to the premature activation of the plantar flexor muscles in stroke subjects referred by Den Otter et al. (2007).

In the present study, the lower EMGa of TA in CONTRA limb is in agreement with the results of the study described in (Achache et al., 2010), which is compatibility with a lateral cortico-spinal system dysfunction. Also, the lower values of SOL EMGa in the IPSI limb may be explained by the dysfunction in the ventro-medial system. Consequently, the non existence of statistical significant differences between the two limbs may be justified by the referred bilateral system dysfunction and the influence of the agonist over the antagonist muscles. Some studies found no significant differences between the two limbs in relation to strength (Hsu et al., 2002; Milot et al., 2006), however there is little evidence about the possibility of a bilateral dysfunction. Although suggesting that both limbs are affected, the results of this study need further research, including participants without neurologic impairment. Previews studies demonstrated that stroke subjects presents lower SOL EMGa in IPSI limb when compared to the healthy subjects in initial phase of standing to sitting (Silva et al., 2012).

We hypothesized that a possible dysfunction of the ventro-medial system, with repercussions over SOL in IPSI limb, that results from a lesion in cortico-reticular system, with repercussion over TA in CONTRA limb, can explain the lower SOL EMGa in IPSI limb. In another perspective, Lamontagne et al. (2002) explains the weakness in IPSI limb as a result of an excessive coativation of the plantarflexor and the dorsiflexor muscles. It is important to note that in midstance (were SOL acts as an agonist) higher levels of the antagonist $\mathrm{C}$ were observed in the IPSI limb. This may be because, in this limb, the decreased SOL EMGa led to lower levels of reciprocal inhibition over to TA. In spite of the non existence of statistical significant differences in the antagonist $\mathrm{C}$ between limbs in initial contact and terminal stance, the differences observed in mean values seems to be associated to the lack of modulation of agonist through antagonist muscles.

The neuronal connection of SOL and TA need further discussion, but it can constitute a possible explanation to the dysfunction in the IPSI limb in stroke subjects observed in some studies (Lamontagne, et al., 2002; Peterson et al., 2010). In fact, it is highly dependent of the Ib and II afferent fibers to modulate the activity of the SOL muscle (Mazzaro et al., 2007), which can in turn influence the activity of TA by the reciprocal inhibition mechanism. This hypothesis aloud to see the stroke subjects with a possible problem of postural control in the IPSI limb, and a problem of movement in the CONTRA limb. These results also seem to indicate that the midstance of gait might be the one that reveals most evident neuronal changes of the IPSI limb to the side of the lesion. How these two problems can explain the gait pattern in stroke subjects needs further discussion, in order to understand the possible influence of one limb (with respective problem) over the other. Indeed, there is evidence that gastrocnemius muscle activity 
during propulsion of one limb is related to the magnitude of ground forces during the heel strike of the other limb (Sousa et al., 2012).

The results of this study suggest that we must consider the dysfunction of the neuronal system over the IPSI limb in stroke subjects with repercussions over the antagonist $C$ level in midstance of gait. This finding contradicts the idea that changes in the IPSI limb results from a simple adaptation through the decreased activity in the CONTRA limb (Lamontagne, et al., 2002). Consequently, the therapeutic decisions must consider the need to promote a better postural control of the IPSI limb and do not only be focused on the CONTRA limb. The understanding of these results can be complemented by the study of the role of SOL in IPSI limb in forward propulsion of the trunk as this muscle is the primary contributor to this function (Neptune, et al., 2001).

\section{REFERENCES}

Achache V., Mazevet D., Iglesias C., Lackmy A., Nielsen J. B., Katz R., et al. 2010. Enhanced spinal excitation from ankle flexors to knee extensors during walking in stroke patients. Clinical neurophysiology : official journal of the International Federation of Clinical Neurophysiology, 121(6): 930-938.

Ayyappa E. 1997. Normal Human Locomotion, Part 2: Motion, Ground-Reaction Force and Muscle Activity. Journal of Prosthetics and Orthotics, 9(2): 42-57.

Billot M., Simoneau E., Van Hoecke J. \& Martin A. 2010. Coactivation at the ankle joint is not sufficient to estimate agonist and antagonist mechanical contribution. Muscle \& Nerve, 41(4): 511-518.

Bonell C., Tabernig C. \& Tabernig C. (2007). Analysis of EMG temporal parameters from the tibialis anterior during hemiparetic gait. Paper presented at the 16th Argentine Bioengineering Congress and the 5th Conference of Clinical Engineering.

Busse M., Wiles C. \& van Deursen R. 2006. Co-activation: its association with weakness and specific neurological pathology. Journal of NeuroEngineering and Rehabilitation, 3(1): 26.

Capaday C., Lavoie B. A., Barbeau H., Schneider C. \& Bonnard M. 1999. Studies on the Corticospinal Control of Human Walking. I. Responses to Focal Transcranial Magnetic Stimulation of the Motor Cortex. Journal of Neurophysiology, 81(1): 129-139.

Cappellini G., Ivanenko Y. P., Poppele R. E. \& Lacquaniti F. 2006. Motor Patterns in Human Walking and Running. Journal of Neurophysiology, 95(6): 3426-3437.

Chmielewski T., Hurd W., Rudolph K., Axe M. \& Snyder-Mackler L. 2005. Perturbation training improves knee kinematics and reduces muscle co-contraction after complete unilateral anterior cruciate ligament rupture. Physical Therapy, 85(8): 740-749.

Darainy M. \& Ostry D. 2008. Muscle cocontraction following dynamics learning. Experimental Brain Research, 190(2): 153-163.

Den Otter A. R., Geurts A. C. H., Mulder T. \& Duysens J. 2007. Abnormalities in the temporal patterning of lower extremity muscle activity in hemiparetic gait. Gait \&amp; Posture, 25(3): 342-352.

Frigo C. \& Crenna P. 2009. Multichannel SEMG in clinical gait analysis: A review and state-ofthe-art. Clinical biomechanics (Bristol, Avon), 24(3): 236-245. 
Higginson J. S., Zajac F. E., Neptune R. R., Kautz S. A. \& Delp S. L. 2006. Muscle contributions to support during gait in an individual with post-stroke hemiparesis. Journal of Biomechanics, 39(10): 1769-1777.

Hsu A.-L., Tang P.-F. \& Jan M.-H. 2002. Test-retest reliability of isokinetic muscle strength of the lower extremities in patients with stroke. Archives of Physical Medicine and Rehabilitation, 83(8): 1130-1137.

Lamontagne A., Malouin F., Richards C. L. \& Dumas F. 2002. Mechanisms of disturbed motor control in ankle weakness during gait after stroke. Gait \&amp; Posture, 15(3): 244-255.

Lamontagne A., Richards C. L. \& Malouin F. 2000. Coactivation during gait as an adaptive behavior after stroke. Journal of Electromyography and Kinesiology, 10(6): 407-415.

Lin P.-Y., Yang Y.-R., Cheng S.-J. \& Wang R.-Y. 2006. The Relation Between Ankle Impairments and Gait Velocity and Symmetry in People With Stroke. Archives of Physical Medicine and Rehabilitation, 87(4): 562-568.

Mayer M. 2002. Clinical neurokinesiology of spastic gait. Bratisl Lek Listy 103(1): 3-11.

Mazzaro N., Nielsen J. F., Grey M. J. \& Sinkjaer T. 2007. Decreased Contribution From Afferent Feedback to the Soleus Muscle During Walking in Patients With Spastic Stroke. Journal of Stroke and Cerebrovascular Diseases, 16(4): 135-144.

Milot M.-H., Nadeau S., Gravel D. \& Bourbonnais D. 2008. Effect of increases in plantarflexor and hip flexor muscle strength on the levels of effort during gait in individuals with hemiparesis. Clinical Biomechanics, 23(4): 415-423.

Milot M.-H., Nadeau S., Gravel D. \& Requião L. F. 2006. Bilateral Level of Effort of the Plantar Flexors, Hip Flexors, and Extensors During Gait in Hemiparetic and Healthy Individuals. Stroke, 37(8): 2070-2075.

Neptune R. R., Kautz S. A. \& Zajac F. E. 2001. Contributions of the individual ankle plantar flexors to support, forward progression and swing initiation during walking. Journal of Biomechanics, 34(11): 1387-1398.

Orendurff M. S., Segal A. D., Aiona M. D. \& Dorociak R. D. 2005. Triceps surae force, length and velocity during walking. Gait \&amp; Posture, 21(2): 157-163.

Peterson C. L., Hall A. L., Kautz S. A. \& Neptune R. R. 2010. Pre-swing deficits in forward propulsion, swing initiation and power generation by individual muscles during hemiparetic walking. Journal of Biomechanics, 43(12): 2348-2355.

Schumway-Cook A. \& Woollacott M. (2007). Motor Control - Translating research into clinical practice ((4th ed).). Philadelphia: Lippincott Williams \& Wilkins.

Silva A., Pinheiro A., Sousa A., Tavares J., Santos R. \& Sousa F. 2012. Soleus activity in poststroke subjects: movement sequence from standing to sitting. Somatossensory and Motor Research, (in press).

Sousa A. S., Santos R., Oliveira F. P., Carvalho P. \& Tavares J. M. R. 2012. Analysis of ground reaction force and electromyographic activity of the gastrocnemius muscle during double support. Proceedings of the Institution of Mechanical Engineers, Part H: Journal of Engineering in Medicine. 
FIGURES
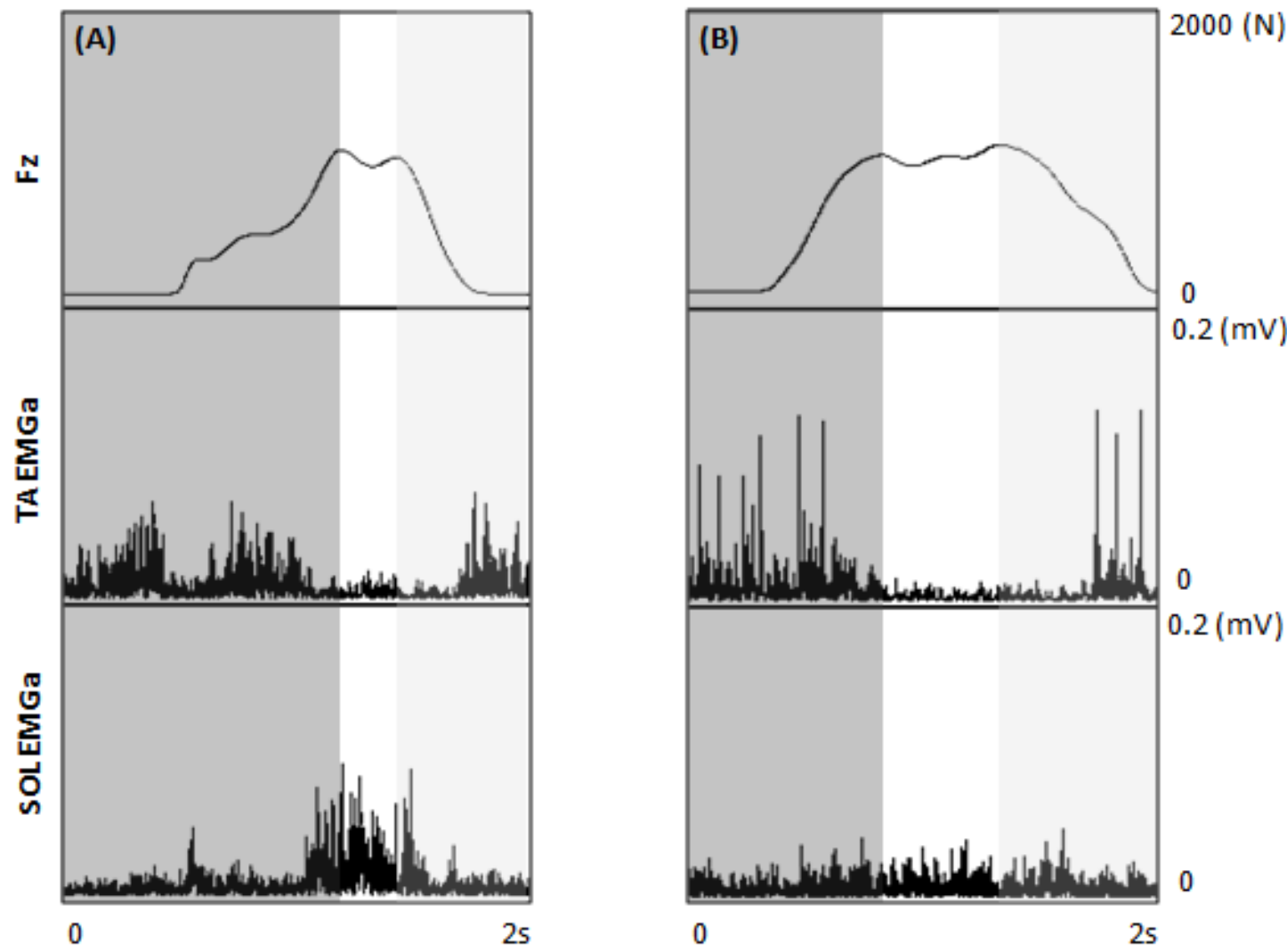

Figure 1: Example of the raw EMG of TA and SOL muscles and of the Fz acquired in this work during the stance phase of gait in IPSI CONTRA (A) and CONTRA limb (B). 


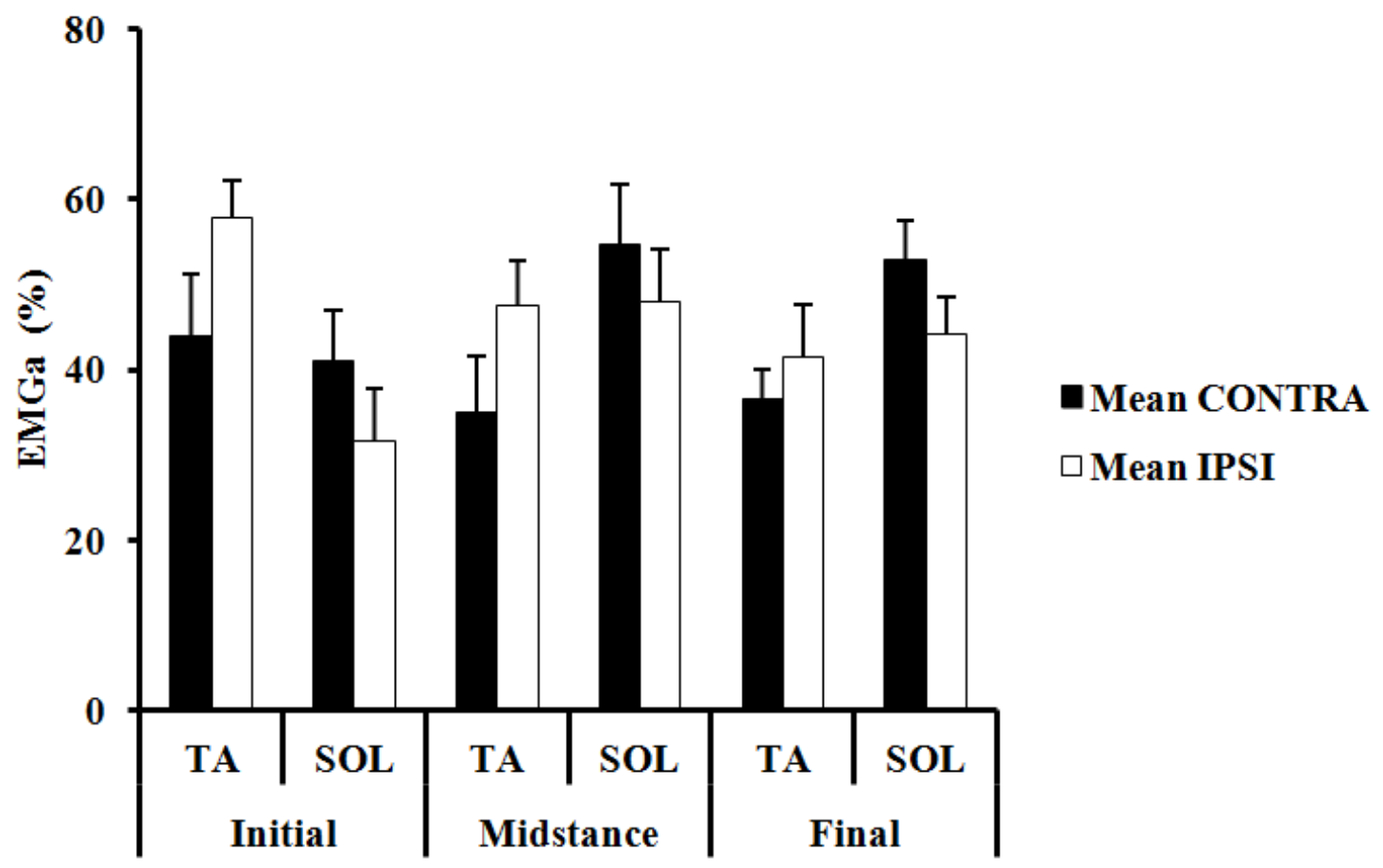

Figure 2: Relative EMGa (\%) of TA and SOL during stance phase of gait in the IPSI and the CONTRA limb in relation to the side of the lesion. 
TABLES

Table 1: Mean (M) and standard deviation (SD) values of age, height and weight, and the time evolution and identification of the lesion side, of the stroke subjects studied.

\begin{tabular}{lcc}
\hline \multicolumn{1}{c}{ Variable } & \multicolumn{2}{c}{ Stroke subjects } \\
& $\mathrm{M}$ & $\mathrm{SD}$ \\
\hline Age (years) & 56.4 & 8.40 \\
Height (cm) & 165.2 & 10.86 \\
Weight (Kg) & 76.8 & 8.81 \\
\hline $\begin{array}{lcc}\text { Clinical evaluation } \\
\text { (months) }\end{array}$ & 26.0 & 10.4 \\
$\begin{array}{l}\text { Lesion side, } \\
\text { number of subjects }\end{array}$ & Right, $\mathrm{n}=3$ & Left, $\mathrm{n}=9$ \\
\hline
\end{tabular}

Table 2: Antagonist $C$ values (\%) obtained during the stance phase of gait in the IPSI and the CONTRA limb in relation to the side of the lesion.

\begin{tabular}{|c|c|c|c|}
\hline \multirow{2}{*}{ Stance subphases } & \multicolumn{2}{|c|}{ Mean (SD) } & \multirow{2}{*}{ Proof value } \\
\hline & CONTRA & IPSI & \\
\hline Initial contact & $48.9(22.0)$ & $42.2(21.2)$ & 0.209 \\
\hline Midstance & 38.4 (13.6) & $52.6(13.0)$ & $0.015^{*}$ \\
\hline Final stance & $41.4(13.4)$ & $47.4(12.5)$ & 0.272 \\
\hline
\end{tabular}

